$16^{\text {th }}$ International Conference on

AEROSPACE SCIENCES \& AVIATION TECHNOLOGY,

ASAT - 16 - May 26 - 28, 2015, E-Mail: asat@ mtc.edu.eg

Military Technical College, Kobry Elkobbah, Cairo, Egypt

Tel : +(202) $24025292-24036138$, Fax: +(202) 22621908

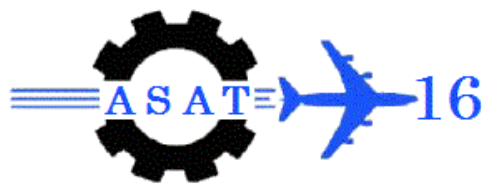

\title{
Analysis and Design of UWB Modified Two-Sections Wilkinson Power Splitter
}

\author{
Shaimaa A. Osman*, Ayman M. El-Tager ${ }^{\dagger}$, Fawzy Ibrahim ${ }^{\ddagger}$ and I. M. Hafez ${ }^{\S}$
}

\begin{abstract}
In this paper, the analysis and design of a compact Ultra-Wideband (UWB) Wilkinson Power Splitter (WPS) is presented. The proposed splitter consists of two sections Wilkinson power splitter of equal characteristic impedances and unequal electrical lengths with a single stub on each section. The proposed power splitter examined analytically using the "Even Odd Mode" analysis method and the ABCD matrix. The simulation is done using Agilent ADS circuit and EM simulator. The proposed structure achieves an input return loss, $\mathrm{S}_{11}$ better than $12.2 \mathrm{~dB}$, an output return loss, $\mathrm{S}_{22}$ and $\mathrm{S}_{33}$ better than $19.59 \mathrm{~dB}$, output ports isolation, $S_{23}$ and $S_{32}$ better than $12.75 \mathrm{~dB}$ and insertion loss, $S_{21}$ and $S_{31}$ from 3.15 to $3.41 \mathrm{~dB}$ through the whole UWB range from $3.1 \mathrm{GHz}$ to $10.6 \mathrm{GHz}$. Furthermore, the power splitter has a compact size of $18.38 * 10.25 \mathrm{~mm}^{2}$ compared to other similar networks.
\end{abstract}

Keywords: UWB Wilkinson Power Splitter, Divider, Single stub, Even-Odd, ABCD Matrix, Isolation Enhancement.

\section{INTRODUCTION}

Power dividers and combiners are essential passive microwave components. Wilkinson Power Divider (WPD)[1] shown in Fig.1 is the most practical and popular type as it provides matched ports, good isolation between output ports, lossless even when output ports are matched; only reflected power is dissipated [2] also, equal ratio WPD can be easily analyzed using the "Even-odd Mode Method" [3].

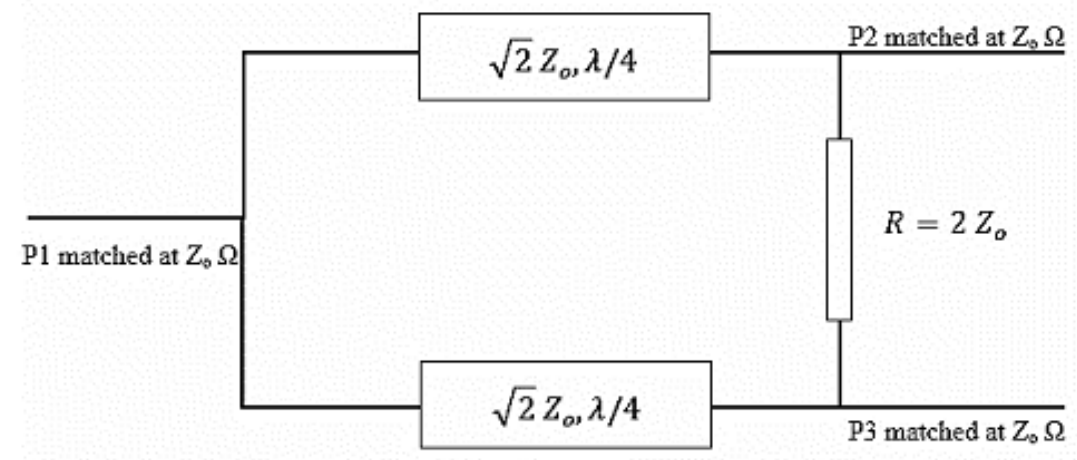

Fig. 1. Conventional Transmission line WPD.

\footnotetext{
*Egyptian Russian University (ERU), shaimaa.osman@yahoo.com.

${ }^{\dagger}$ Military Technical College (MTC).

${ }^{\ddagger}$ Misr International University (MIU).

${ }^{4}$ Ain Shams University.
} 
The Federal Communication Commission (FCC) of USA has assigned the band $3.1 \mathrm{GHz}$ to 10.6 GHz for Ultra-Wideband (UWB), which is a short-range wireless communication technology for transmitting high-speed data using extremely low power. The narrow bandwidth of WPD is a problem for UWB applications, recently many designs tried to overcome bandwidth limitation of WPD for UWB, also tried to improve the ports isolation and output matching. Exponential tapered line replaced the quarter wavelength transmission line in [4] and [5] to improve the S-parameters and the bandwidth but on expenses of using multiple lumped elements and having large area. In [6] an open stub and parallel-coupled lines are installed to output ports, the design achieves moderate S-parameters. In [7] a design for UWB WPD with delta stub is introduced, Delta stub is good alternative for radial stub as it has simpler contour and it is easier to be fabricated, the design has small area and moderate $\mathrm{S}$ parameters.

In [8] an UWB power divider consists of two microstrip transmission lines, parallel-coupled microstrip transmission line and a single resistor introduced. The design is very compact, single layer and has two transmission poles at $5.2 \mathrm{GHz}$ and $9.5 \mathrm{GHz}$; it gives moderate simulation parameters along the UWB Range. In [9] an UWB power divider consists of Stepped-impedance three-line coupled structure and microstrip to slot-line transitions is introduced; it achieves good S-parameters with moderate area. In [10] single, two and three sections Wilkinson power divider using binomial multi-section matching transformer is designed for ultra-wideband applications, good S-parameters are achieved for two and three sections.

When designing an UWB Wilkinson power divider (WPD), combiner or splitter, three main criteria must be taken into consideration; isolation enhancement, matching improvement and bandwidth broadening. Isolation enhancement can be achieved using multiple sections of WPD but in most cases on expense of large area. In [11] series and parallel RLC circuit are used to realize complex isolation component, but LC are not recommended in microwave frequencies as it gives a narrow bandwidth. In [12] a dual frequency power divider with isolation stubs is presented where stubs are used to connect the isolation resistor; this technique achieves a good isolation at the dual frequencies. Matching improvement and bandwidth broadening are achieved mainly using either single stub matching network as in [7], [13] and [14], double stub matching network as in [15] or triple stub matching network as in [16].

In this paper, a compact UWB modified Wilkinson Power Splitter (WPS) is analyzed and implemented. The rest of this paper is organized as: Section II presents two analytical approaches: even-odd mode analysis method and ABCD matrix technique. Section III introduces the design procedure to calculate initial design values that will be used in simulation. Section IV illustrates the resulting simulated S-parameters for the transmission line representation and Section V discusses the implementation of the splitter, results analysis and comparison with other similar implementations. 


\section{ANALYSIS OF THE PROPOSED MODIFIED WPS}

The schematic diagram of the proposed UWB modified Wilkinson power splitter is shown in Fig. 2. It consists of two sections WPS each of a transmission line followed by a single stubmatching network and a single isolation resistor. The stub matching networks installed to broaden the bandwidth and to improve the matching of the splitter. The isolation improved by using two sections of "WPS with single stub". Moreover, to achieve perfect matching with input and output ports, the input and the output transmission lines are chosen to have characteristic impedance, $Z_{0}=50 \Omega$, thus their electrical length will have no effect on the matching.

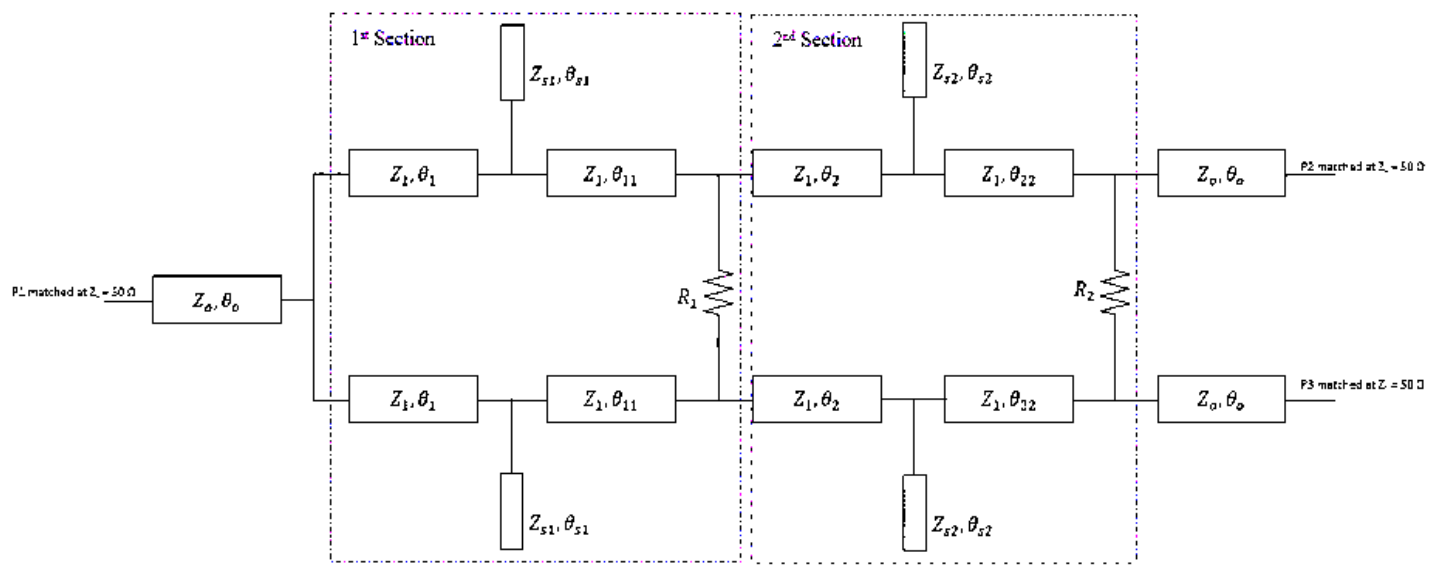

Fig. 2 . Schematic diagram for the proposed UWB modified two sections WPS.

\section{II.1. Even-Odd Analysis}

The proposed power splitter has a symmetric structure, thus it can be analytically analyzed using "Even-Odd Mode" method of Reed and Wheeler [17]. It has been used also by Cohn [3] to analyze Wilkinson Power Divider. Fixing the input and output ports at $\mathrm{Z}_{\mathrm{o}}=50 \Omega$ helps simplifying the analysis by omitting them from the design procedure as illustrated in Fig. 3. In addition, fixing the characteristic impedance of the two sections limits the flexibility of design but simplifies the analytical solution.

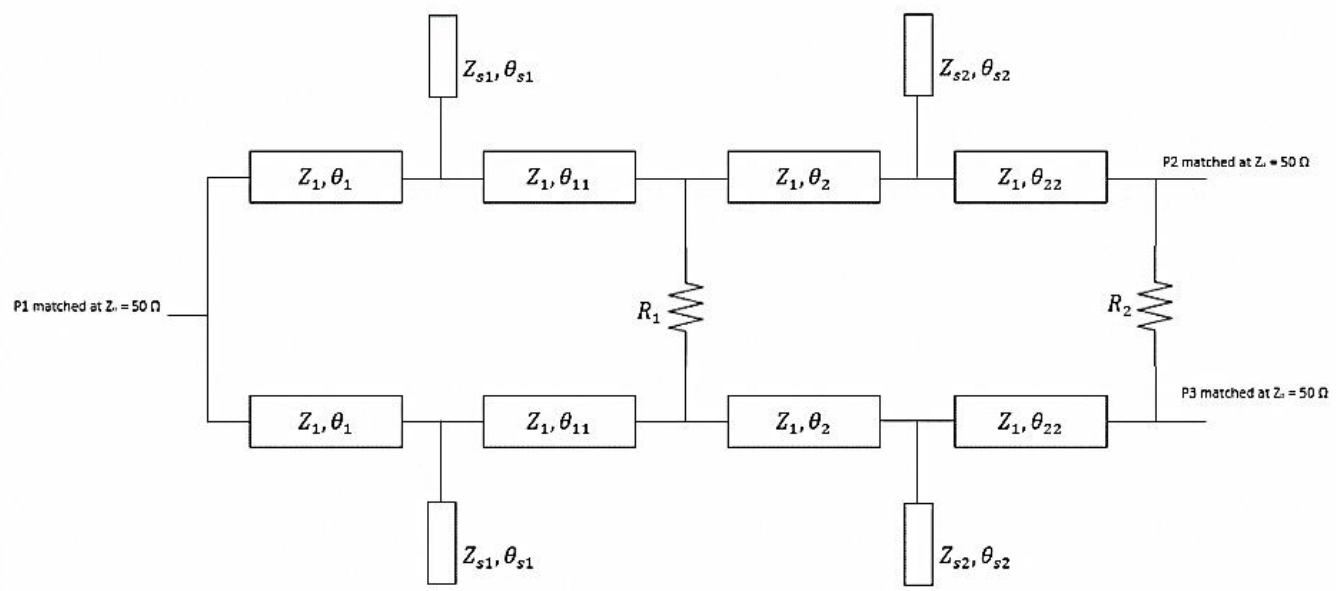

Fig. 3. Simplified schematic diagram after fixing the input and output ports to Zo $=50 \Omega$.

The analysis Starts by replacing each section with its equivalent single transmission line as illustrated in Fig. 4. In Cohn [3] the case of equal electrical length transmission lines is examined, here we examine the case of unequal electrical length transmission lines and equal characteristic impedance i.e. $\varphi_{1} \neq \varphi_{2}, Z_{\text {line } 1}=Z_{\text {line } 2}=Z_{t}$. 


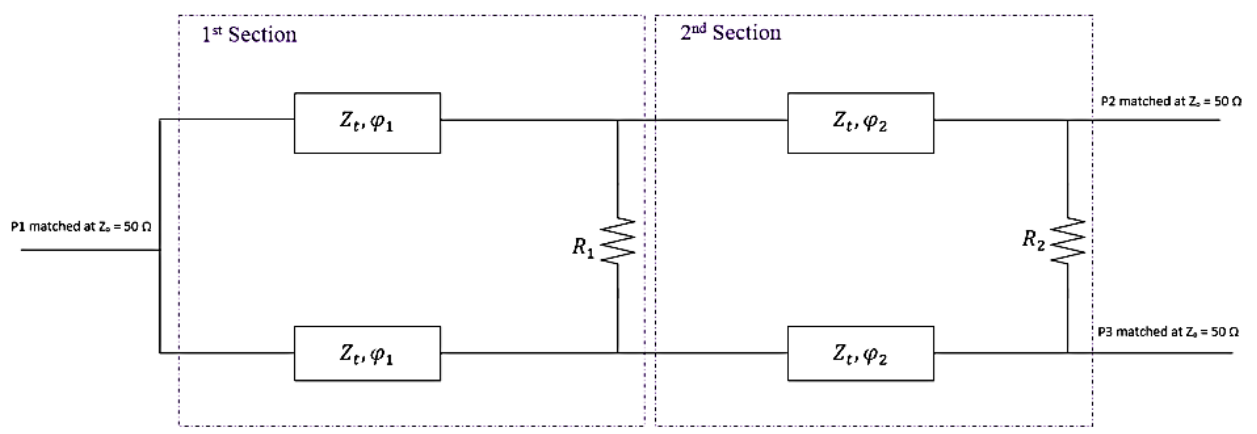

(a)

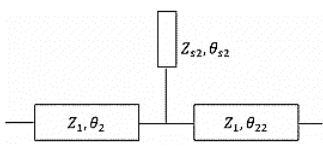

(b)

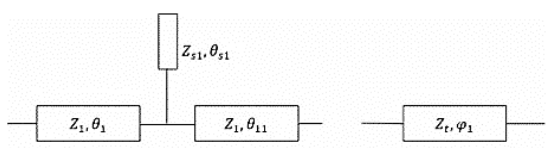

(c)

Fig. 4. (a) Proposed power splitter after simplification, (b) 1st section equivalent, (c) 2nd section equivalent.

1- Even-mode analysis at Port 1 (P1)

With even-mode excitation, an equal magnitude zero phase difference signals are applied to port 2 and port 3 . Therefore, there is no voltage difference across the resistors and the circuit is bisected at the mid-point with open circuit (o.c). i.e. isolation resistors have no effect in even mode analysis. Moreover, the impedance at port 1 doubled, as there is no current flow through the short circuit between the inputs of the two transmission lines at port 1 as in Fig. 5 . It is convenient to use the admittance representation of transmission lines.

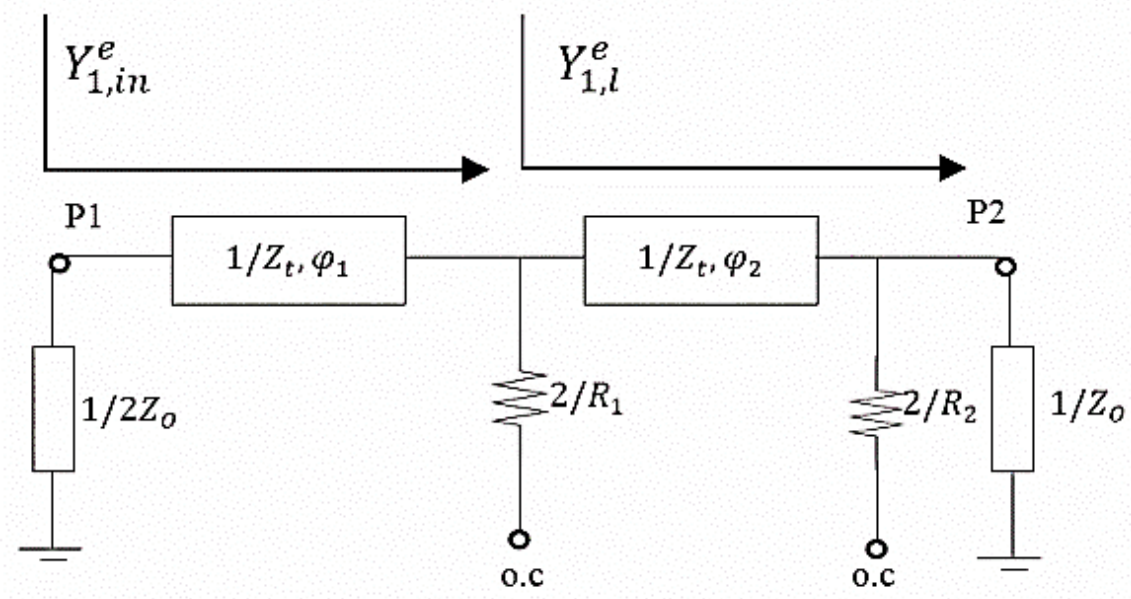

Fig. 5. Even-mode bisection of the proposed splitter in admittance representation, input admittance seen at P1.

For perfect input and output matching:

$$
S_{11}^{e}=S_{22}^{e}=S_{22}^{o}=0
$$

Where $S_{11}^{e}$ is the even-mode scattering parameter at Port $1(\mathrm{P} 1), S_{22}^{e}$ is the even-mode Sparameter at Port $2(\mathrm{P} 2)$ and $S_{22}^{o}$ is the odd-mode S-parameter at P2. Using Cohn's procedure [3] for two sections Wilkinson, the input admittance at $\mathrm{P} 1, Y_{1, i n}^{e}$, can be found from:

$$
Y_{1, i n}^{e}=\frac{1}{Z_{t}} \frac{Y_{1, l}^{e}+j \frac{\tan \varphi_{1}}{Z_{t}}}{\frac{1}{Z_{t}}+j Y_{1, l}^{e} \tan \varphi_{1}}
$$




$$
Y_{1, l}^{e}=\frac{1}{Z_{t}} \frac{\frac{1}{Z_{o}}+j \frac{\tan \varphi_{2}}{Z_{t}}}{\frac{1}{Z_{t}}+j \frac{1}{Z_{o}} \tan \varphi_{2}}
$$

The even-mode scattering parameter at P1: $S_{11}^{e}=\frac{\frac{1}{2 Z_{o}}-Y_{1, i n}^{e}}{\frac{1}{2 Z_{o}}+Y_{1, i n}^{e}}$

From eq. (1) and eq. (4)

$$
Y_{1, i n}^{e}=\frac{1}{2 Z_{o}}
$$

Substitute eq. (5) into eq. (2), we get

$$
\frac{Z_{t}}{2 Z_{o}}=\frac{Z_{t} Y_{1, l}^{e}+j \tan \varphi_{1}}{1+j Z_{t} Y_{1, l}^{e} \tan \varphi_{1}}
$$

$$
Z_{t} Y_{1, l}^{e}=\frac{j 2 Z_{o} \tan \varphi_{1}-Z_{t}}{j Z_{t} \tan \varphi_{1}-2 Z_{o}}
$$

Also, rearrange eq. (3), it becomes

$$
Z_{t} Y_{1, l}^{e}=\frac{Z_{t}+j Z_{o} \tan \varphi_{2}}{Z_{o}+j Z_{t} \tan \varphi_{2}}
$$

Equate eq. (6) and eq. (7) we get

$$
j Z_{t}^{2}\left(\tan \varphi_{1}+\tan \varphi_{2}\right)+Z_{t}\left(-Z_{o}+Z_{o} \tan \varphi_{1} \tan \varphi_{2}\right)-j 2 Z_{o}^{2}\left(\tan \varphi_{1}+\tan \varphi_{2}\right)=0
$$

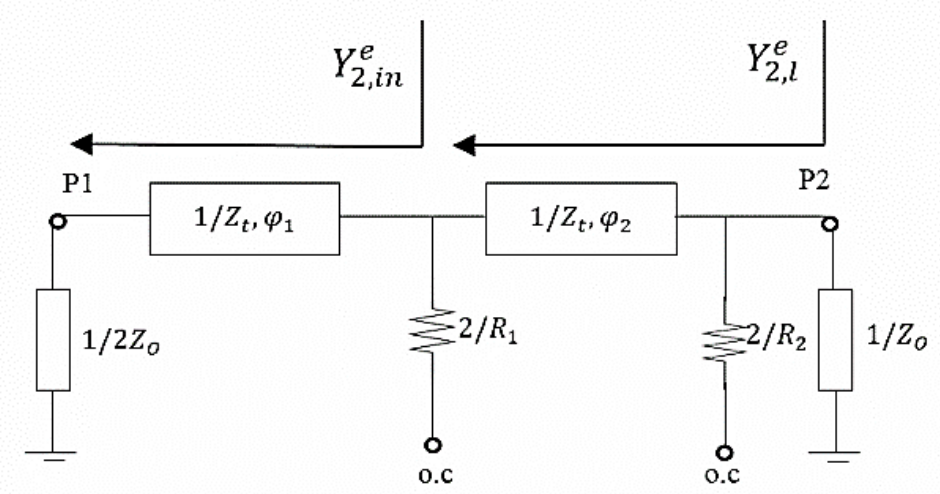

Fig. 6. Even-mode bisection of the proposed splitter in admittance representation, input admittance seen at $\mathbf{P 2}$.

2- Even-mode analysis at Port 2 (P2)

The input admittance at $\mathrm{P} 2, Y_{2, i n}^{e}$, as in Fig. 6 can be found from

$$
Y_{2, \text { in }}^{e}=\frac{1}{Z_{t}} \frac{Y_{2, l}^{e}+j \frac{\tan \varphi_{2}}{Z_{t}}}{\frac{1}{Z_{t}}+j Y_{2, l}^{e} \tan \varphi_{2}}
$$




$$
Y_{2, l}^{e}=\frac{1}{Z_{t}} \frac{\frac{1}{2 Z_{o}}+j \frac{\tan \varphi_{1}}{Z_{t}}}{\frac{1}{Z_{t}}+j \frac{1}{2 Z_{o}} \tan \varphi_{1}}
$$

The even-mode scattering parameter at P2: $\quad S_{22}^{e}=\frac{\frac{1}{Z_{0}}-Y_{2, i n}^{e}}{\frac{1}{Z_{o}}+Y_{2, i n}^{e}}$

From eq. (1) and eq. (11)

$$
Y_{2, \text { in }}^{e}=\frac{1}{Z_{o}}
$$

Substitute eq. (12) into eq. (9), we get

$$
\frac{Z_{t}}{Z_{o}}=\frac{Z_{t} Y_{2, l}^{e}+j \tan \varphi_{2}}{1+j Z_{t} Y_{2, l}^{e} \tan \varphi_{2}}
$$

$$
Z_{t} Y_{2, l}^{e}=\frac{j Z_{o} \tan \varphi_{2}-Z_{t}}{j Z_{t} \tan \varphi_{2}-Z_{o}}
$$

Also, rearrange eq. (10), it becomes

$$
Z_{t} Y_{2, l}^{e}=\frac{Z_{t}+j 2 Z_{o} \tan \varphi_{1}}{2 Z_{o}+j Z_{t} \tan \varphi_{1}}
$$

Equate eq. (13) and eq. (14) we get

$$
j Z_{t}^{2}\left(\tan \varphi_{1}+\tan \varphi_{2}\right)+Z_{t}\left(Z_{o}-Z_{o} \tan \varphi_{1} \tan \varphi_{2}\right)-j 2 Z_{o}^{2}\left(\tan \varphi_{1}+\tan \varphi_{2}\right)=0
$$

From eq. (8) and eq. (15) we get

$$
Z_{t}=\sqrt{2} Z_{o}
$$

3- Odd-mode analysis at Port $2(\mathrm{P} 2)$

With odd-mode excitation, an equal magnitude $180^{\circ}$ phase difference signals are applied to port 2 and port 3 . Therefore, there is a voltage null along the mid-point of the circuit and it can be bisected by using ground as in Fig. 7 .

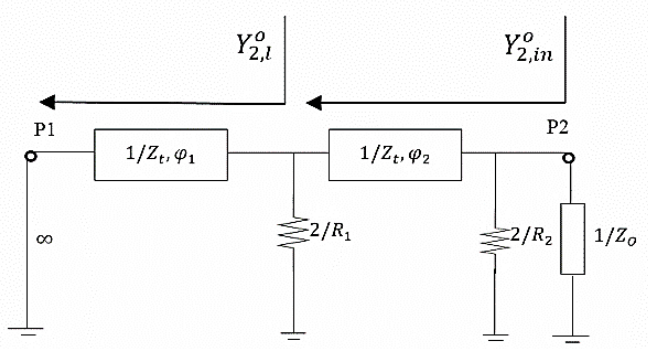

Fig. 7. Odd-mode bisection of the proposed splitter in admittance representation, input admittance seen at P2.

The input admittance at $\mathrm{P} 2, Y_{2, \text { in }}^{o}$, can be found from

$$
Y_{2, \text { in }}^{o}=\frac{2}{R_{2}}+\frac{1}{Z_{t}} \frac{Y_{2, l}^{o}+j \frac{\tan \varphi_{2}}{Z_{t}}}{\frac{1}{Z_{t}}+j Y_{2, l}^{o} \tan \varphi_{2}}
$$




$$
Y_{2, l}^{o}=\frac{2}{R_{1}}-j \frac{1}{Z_{t} \tan \varphi_{1}}
$$

The odd-mode scattering parameter at P2: $S_{22}^{o}=\frac{\frac{1}{Z_{o}}-Y_{2, \text { in }}^{o}}{\frac{1}{Z_{o}}+Y_{2, \text { in }}^{o}}$

From eq. (1) and eq. (19)

$$
Y_{2, i n}^{o}=\frac{1}{Z_{o}}
$$

Substitute eq. (20) into eq. (17), we get

$$
\begin{array}{r}
\frac{1}{Z_{o}}-\frac{2}{R_{2}}=\frac{1}{Z_{t}} \frac{Z_{t} Y_{2, l}^{o}+j \tan \varphi_{2}}{1+j Z_{t} Y_{2, l}^{o} \tan \varphi_{2}} \\
Z_{t} Y_{2, l}^{o}=\frac{j Z_{o} R_{2} \tan \varphi_{2}-Z_{t} R_{2}+2 Z_{o} Z_{t}}{j Z_{t} R_{2} \tan \varphi_{2}-j 2 Z_{o} Z_{t} \tan \varphi_{2}-Z_{o} R_{2}} \ldots \ldots \ldots \ldots \ldots \ldots \ldots \ldots \ldots \ldots \ldots \ldots \ldots \ldots
\end{array}
$$

Also, rearrange eq. (18), it becomes

$$
Z_{t} Y_{2, l}^{o}=\frac{2 Z_{t} \tan \varphi_{1}-j R_{1}}{R_{1} \tan \varphi_{1}}
$$

Equate eq. (21) and eq. (22) we get

$$
\begin{aligned}
j Z_{o} R_{1} R_{2} \tan \varphi_{1} & \tan \varphi_{2}-Z_{t} R_{1} R_{2} \tan \varphi_{1}+2 Z_{o} Z_{t} R_{1} \tan \varphi_{1} \\
& =j 2 Z_{t}^{2} R_{2} \tan \varphi_{1} \tan \varphi_{2}-j 4 Z_{o} Z_{t}^{2} \tan \varphi_{1} \tan \varphi_{2}-2 Z_{o} Z_{t} R_{2} \tan \varphi_{1}+Z_{t} R_{1} R_{2} \tan \varphi_{2} \\
& -2 Z_{o} Z_{t} R_{1} \tan \varphi_{2}+j Z_{o} R_{1} R_{2} \ldots \ldots \ldots \ldots \ldots \ldots \ldots \ldots \ldots \ldots \cdots \cdots \cdots \cdots \cdots \cdots \cdots(23)
\end{aligned}
$$

Separating the real and the imaginary parts of eq. (23), first, work with the real part to get a formula for the first isolation resistor $\mathrm{R}_{1}$ we get:

$$
R_{1}=\frac{2 Z_{o} R_{2} \tan \varphi_{1}}{R_{2}\left(\tan \varphi_{1}+\tan \varphi_{2}\right)-2 Z_{o}\left(\tan \varphi_{1}+\tan \varphi_{2}\right)}
$$

Second, work with the imaginary part to get a formula for the second isolation resistor $\mathrm{R}_{2}$, where $R_{1}$ is substituted from eq. (23) and $Z_{t}$ is substituted from eq. (16) to get

$$
R_{2}=\frac{4 Z_{o} \tan \varphi_{2}\left(\tan \varphi_{1}+\tan \varphi_{2}\right)+2 \sqrt{2} Z_{o} \sqrt{\tan \varphi_{2}\left(\tan \varphi_{1}+\tan \varphi_{2}\right)} \sqrt{\tan \varphi_{1} \tan \varphi_{2}-1}}{2 \tan \varphi_{2}\left(\tan \varphi_{1}+\tan \varphi_{2}\right)+\left(1-\tan \varphi_{1} \tan \varphi_{2}\right)} \ldots
$$

\section{II.2 ABCD Matrix Analysis}

In order to find the equivalency between the parameters of simplified structure [ $\left.\left(Z_{t}, \varphi_{1}\right),\left(Z_{t}, \varphi_{2}\right)\right]$ of Fig.4 and the detailed one $\left[\left(Z_{1}, \theta_{1}, \theta_{11}\right.\right.$ and $\left.Z_{s 1}, \theta_{s 1}\right),\left(Z_{1}, \theta_{2}, \theta_{22}\right.$ and $\left.\left.Z_{s 2}, \theta_{s 2}\right)\right]$ of Fig.3, the ABCD matrix is used. Starting with the first section of Fig.8 where the shunt open circuit stub admittance $Y_{s 1}$ represented as follows:

$$
\begin{aligned}
& Y_{s 1}=j B_{1}=j \frac{\tan \theta_{s 1}}{Z_{s 1}}, \quad B_{1} \text { is the stub susceptance for the first section } \\
& M_{1} M_{2} M_{3}=\left[\begin{array}{cc}
\cos \varphi_{1} & j Z_{t} \sin \varphi_{1} \\
j \frac{\sin \varphi_{1}}{Z_{t}} & \cos \varphi_{1}
\end{array}\right]
\end{aligned}
$$




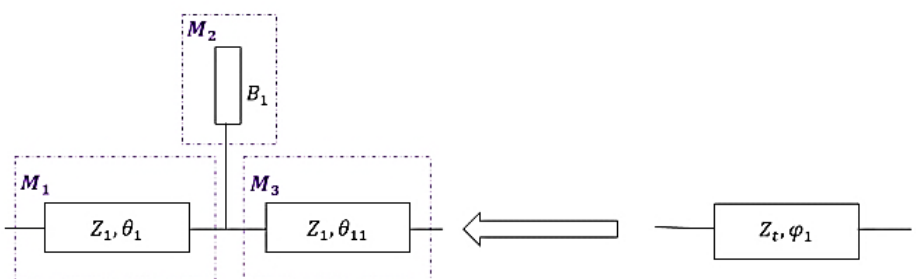

Also,

Fig. 8. Matrices identification of the 1st section

$$
\begin{aligned}
M_{1} M_{2} M_{3} & =\left[\begin{array}{cc}
\cos \theta_{1} & j Z_{1} \sin \theta_{1} \\
j \frac{\sin \theta_{1}}{Z_{1}} & \cos \theta_{1}
\end{array}\right]\left[\begin{array}{cc}
1 & 0 \\
j B_{1} & 1
\end{array}\right]\left[\begin{array}{cc}
\cos \theta_{11} & j Z_{1} \sin \theta_{11} \\
j \frac{\sin \theta_{11}}{Z_{1}} & \cos \theta_{11}
\end{array}\right] \\
& =\left[\begin{array}{cc}
\cos \theta_{1}-Z_{1} B_{1} \sin \theta_{1} & j Z_{1} \sin \theta_{1} \\
j \frac{\sin \theta_{1}}{Z_{1}}+j B_{1} \cos \theta_{1} & \cos \theta_{1}
\end{array}\right]\left[\begin{array}{cc}
\cos \theta_{11} & j Z_{1} \sin \theta_{11} \\
j \frac{\sin \theta_{11}}{Z_{1}} & \cos \theta_{11}
\end{array}\right]
\end{aligned}
$$

Which leads to:

$$
\begin{aligned}
& {\left[\begin{array}{cc}
\cos \left(\theta_{1}+\theta_{11}\right)-Z_{1} B_{1} \sin \theta_{1} \cos \theta_{11} & j Z_{1} \sin \left(\theta_{1}+\theta_{11}\right)-j Z_{1}^{2} B_{1} \sin \theta_{1} \sin \theta_{11} \\
\frac{j \sin \left(\theta_{1}+\theta_{11}\right)}{Z_{1}}+j B_{1} \cos \theta_{1} \cos \theta_{11} & \cos \left(\theta_{1}+\theta_{11}\right)-Z_{1} B_{1} \cos \theta_{1} \sin \theta_{11}
\end{array}\right]} \\
& =\left[\begin{array}{cc}
\cos \varphi_{1} & j Z_{t} \sin \varphi_{1} \\
j \frac{\sin \varphi_{1}}{Z_{t}} & \cos \varphi_{1}
\end{array}\right]
\end{aligned}
$$

Equating element $\mathrm{A}$ of both matrices and arrange for a solution for $\mathrm{B}_{1}$ we get:

$$
B_{1}=\frac{\cos \left(\theta_{1}+\theta_{11}\right)-\cos \varphi_{1}}{Z_{1} \sin \theta_{1} \cos \theta_{11}}
$$

Similarly, equate element $\mathrm{C}$ of both matrices and solve for $\mathrm{Z}_{1}$ :

$$
\frac{\sin \varphi_{1}}{Z_{t}}=\frac{\sin \left(\theta_{1}+\theta_{11}\right)}{Z_{1}}+B_{1} \cos \theta_{1} \cos \theta_{11}
$$

Substitute for $B_{1}$ from eq. (29) and arrange we get that

$$
Z_{1}=Z_{t} \frac{\cos \theta_{11}-\cos \varphi_{1} \cos \theta_{1}}{\sin \varphi_{1} \sin \theta_{1}}, \quad Z_{t}=\sqrt{2} Z_{o}
$$

Repeating the ABCD matrix procedure for the second section of Fig. 9 where the shunt open circuit stub admittance $\mathrm{Y}_{\mathrm{s} 2}$ represented as follows:

$$
\begin{aligned}
& Y_{s 2}=j B_{2}=j \frac{\tan \theta_{s 2}}{Z_{s 2}}, B_{2} \text { is the stub susceptance for the second section } \\
& B_{2}=\frac{\cos \left(\theta_{2}+\theta_{22}\right)-\cos \varphi_{2}}{Z_{1} \sin \theta_{2} \cos \theta_{22}}
\end{aligned}
$$




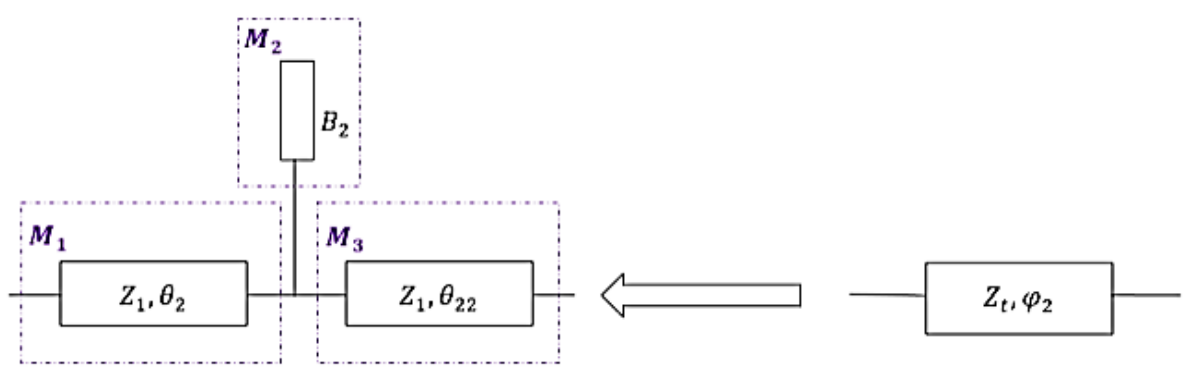

Fig. 9. Matrices identification of the 2 nd section.

\section{INITIAL DESIGN}

In the previous section, a detailed analytical solution for the proposed two sections unequal electrical length power splitter is introduced. This analytical solution is used in this section to get an initial numerical solution for the proposed power splitter according to the following procedures:

1. Determine the characteristic impedance of each section $\left(Z_{\text {line } 1}=Z_{\text {line } 2}=Z_{t}\right)$ :

For input and output ports matched at $Z_{0}=50 \Omega$, then from Eq. (16), the characteristic impedance is given by $Z_{t}=\sqrt{2} Z_{o}=70.71 \Omega$.

2. Determine the electrical length of each section $\left(\varphi_{1}, \varphi_{2}\right)$ :

The conventional Wilkinson power splitter has a length of quarter wavelength $(\lambda / 4)$ for each section i.e. $\varphi=\pi / 2$, that for two sections WPS the total electrical length $\varphi_{\mathrm{t}}=\varphi_{1}+\varphi_{2}=\pi$. Cohn [3] and others had studied the case of equal electrical length sections. Here, in this proposed paper the case of $\varphi_{1}=2 \varphi_{2}$ will be examined. i.e. $\varphi_{1}=120^{\circ}$, then $\varphi_{2}=60^{\circ}$.

3. Calculate the isolation resistors $\left(\mathrm{R}_{1}, \mathrm{R}_{2}\right)$ :

Using Eq. (25), then $\mathrm{R}_{2} \approx 169 \Omega$, substitute in Eq. (24), then $\mathrm{R}_{1} \approx 122 \Omega$.

4. Determine the physical parameters of the $1^{\text {st }}$ section:

The first main transmission line has an electrical length $\left(\theta_{1}\right)$ of quarter wavelength leaving $\lambda / 12$ electrical length divided equally between the stub-matching network $\left(\theta_{11}\right.$, $\theta_{\mathrm{s} 1}$ ), i.e. $\theta_{1}=90^{\circ}, \theta_{11}=15^{\circ}$ and $\theta_{\mathrm{s} 1}=15^{\circ}$. Using eq. (30), the characteristic impedance of the main transmission line $Z_{1}=78.87 \Omega$. From eq. (26) and eq. (29), the stub characteristic impedance $\mathrm{Z}_{\mathrm{s} 1}=84.63 \Omega$.

5. Determine the physical parameters of the $2^{\text {nd }}$ section:

Since the electrical length of the second section is half that of the first section, then $\theta_{2}=$ $45^{\circ}, \theta_{22}=7.5^{\circ}$ and $\theta_{\mathrm{s} 2}=7.5^{\circ}$. Using eq. (31) and eq. (32), the stub characteristic impedance $\mathrm{Z}_{\mathrm{s} 2}=66.92 \Omega$.

\section{CIRCUIT SIMULATION AND OPTIMIZATION}

To examine the performance of the proposed UWB modified WPS, the initial design parameters calculated in Section III are the inputs for the simulator. The Agilent advanced Design System (ADS2011.10) simulation package is used in this work. Tuning and optimization are carried out in order to improve the isolation between the output ports and to broaden the bandwidth. The schematic diagram for the proposed divider after tuning and optimization shown in Fig. 10.

The calculated and optimized parameters of the splitter's ideal transmission line (TL) representation are listed in Table 1. The circuit simulated S-Parameters for the proposed splitter shown in Fig. 11. From the optimized simulation, the following specifications are 
achieved: the input return loss, $\mathrm{S}_{11}$ is better than $12.96 \mathrm{~dB}$, the output return loss, $\mathrm{S}_{22}$ is better than $15.4 \mathrm{~dB}$, the output ports isolation, $S_{23}$ is better than $15 \mathrm{~dB}$ and the insertion loss, $S_{21}$ or $\mathrm{S}_{31}$ ranges from 3.03 to $3.17 \mathrm{~dB}$ through the whole UWB frequency from $3.1 \mathrm{GHz}$ to 10.6 $\mathrm{GHz}$.

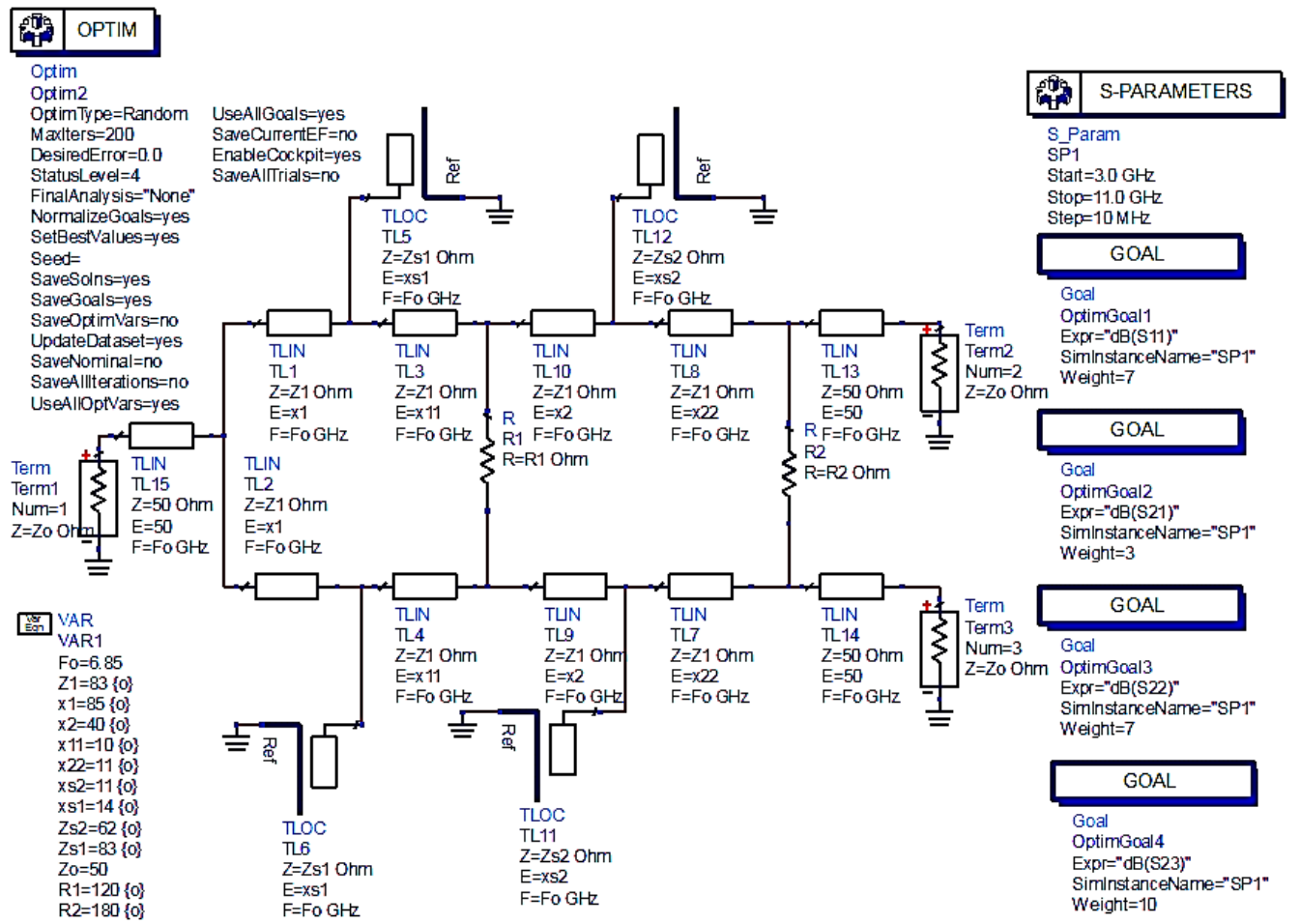

Fig. 10. The Schematic diagram of the ideal TL form for the proposed splitter after optimization

Table 1. Calculated and optimized initial parameters for the proposed ideal TL splitter.

\begin{tabular}{|c|c|c|c|c|c|c|c|c|c|c|c|c|c|}
\hline & \multicolumn{2}{|c|}{$\begin{array}{l}\text { Input and } \\
\text { Output } \\
\text { ports }\end{array}$} & \multicolumn{6}{|c|}{$1^{\text {st }}$ Section } & \multicolumn{5}{|c|}{$2^{\text {nd }}$ Section } \\
\hline & $\mathbf{Z}_{\mathbf{o}}$ & $\boldsymbol{\theta}_{\boldsymbol{o}}$ & $\mathbf{Z}_{1}$ & $\theta_{1}$ & $\theta_{11}$ & $\mathbf{Z}_{\mathrm{s} 1}$ & $\theta_{s 1}$ & $\mathbf{R}_{1}$ & $\boldsymbol{\theta}_{2}$ & $\boldsymbol{\theta}_{22}$ & $\mathbf{Z}_{\mathrm{s} 2}$ & $\theta_{s 2}$ & $\mathbf{R}_{2}$ \\
\hline Calculated & $\begin{array}{l}50 \\
\Omega\end{array}$ & $50^{\circ}$ & $\begin{array}{c}78.87 \\
\Omega\end{array}$ & $90^{\circ}$ & 15 & $\begin{array}{c}84.63 \\
\Omega\end{array}$ & $15^{\circ}$ & $\begin{array}{c}122 \\
\Omega\end{array}$ & $45^{\circ}$ & $7.5^{\circ}$ & $\begin{array}{c}66.92 \\
\Omega\end{array}$ & $7.5^{\circ}$ & $\begin{array}{c}169 \\
\Omega\end{array}$ \\
\hline Optimized & $\begin{array}{l}50 \\
\Omega\end{array}$ & $50^{\circ}$ & $83 \Omega$ & $85^{\circ}$ & $10^{\circ}$ & $83 \Omega$ & $14^{\circ}$ & $\begin{array}{c}120 \\
\Omega\end{array}$ & $40^{\circ}$ & $11^{\circ}$ & $62 \Omega$ & $11^{\circ}$ & $\begin{array}{c}180 \\
\Omega\end{array}$ \\
\hline
\end{tabular}




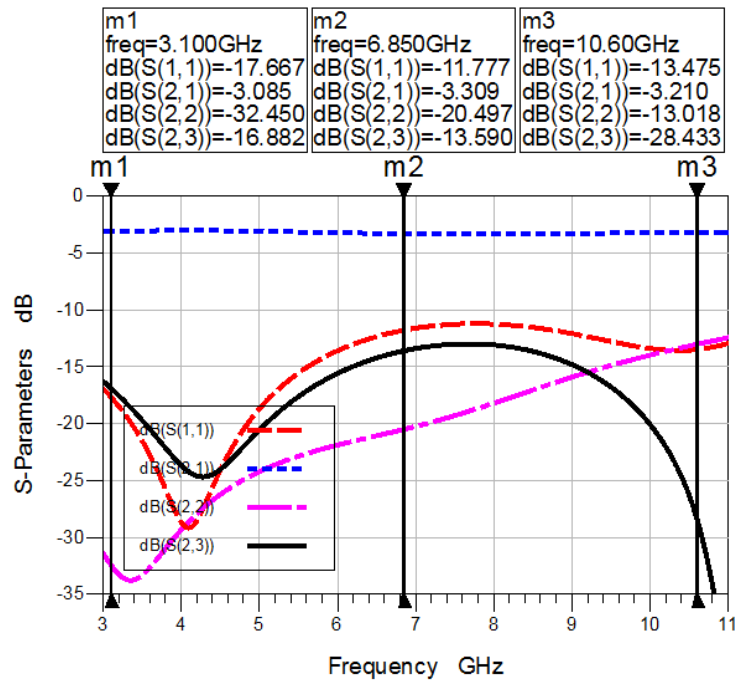

(a)

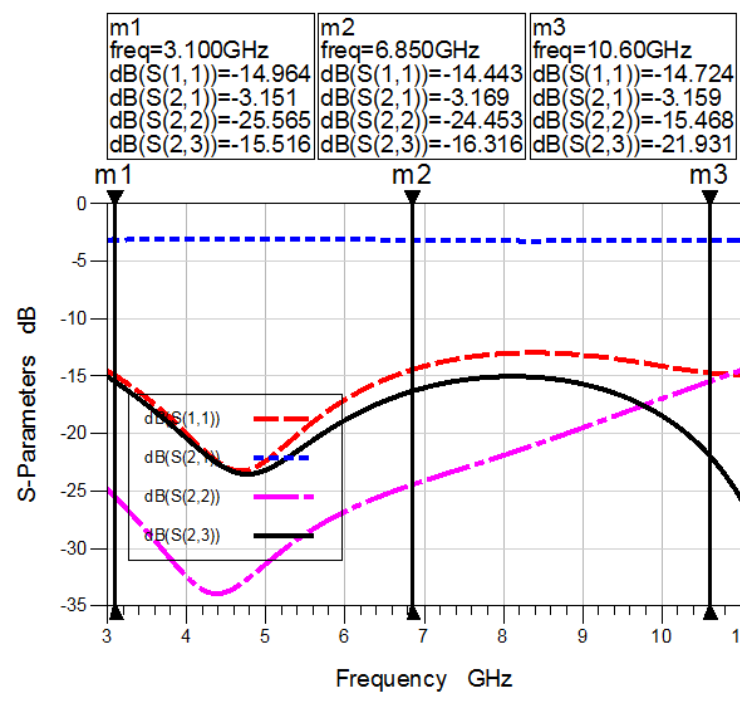

(b)

Fig. 11. The circuit Simulation S-Parameters for the proposed TL form of the Splitter according to: (a) Initial calculation, (b) Tuning and optimization.

\section{IMPLEMENTATION}

The proposed UWB modified WPS realized using Rogers_RT_Duroid 5880 substrate. The substrate parameters are: the dielectric constant, $\varepsilon_{r}=2.2$, loss tangent Tan $\delta=0.0009$, height, $\mathrm{h}=0.787 \mathrm{~mm}$ and conductor thickness, $\mathrm{t}=17 \mu \mathrm{m}$. The final dimensions of the design calculated with the aid of LineCalc tool on Agilent ADS are listed in Table 2. The final schematic layout diagram is shown in Fig.12.

Table 2. Final dimension for the proposed microstrip splitter.

\begin{tabular}{|c|c|c|c|c|c|c|c|c|c|c|}
\hline \multicolumn{2}{|c|}{$\begin{array}{c}\text { Input and } \\
\text { output ports }\end{array}$} & \multicolumn{5}{|c|}{$1^{\text {st }}$ Section } & \multicolumn{4}{|c|}{$2^{\text {nd }}$ Section } \\
\hline $\begin{array}{c}\mathrm{W}_{\mathrm{O}} \\
(\mathrm{mm})\end{array}$ & $\begin{array}{c}\mathrm{L}_{\mathrm{o}} \\
(\mathrm{mm})\end{array}$ & $\begin{array}{l}\mathrm{W}_{1} \\
(\mathrm{~mm})\end{array}$ & & $\mathrm{L}_{11}$ & $\begin{array}{l}\mathrm{W}_{\mathrm{s} 1} \\
(\mathrm{~mm})\end{array}$ & $\begin{array}{l}\mathrm{L}_{\mathrm{s} 1} \\
(\mathrm{~mm})\end{array}$ & & $\begin{array}{l}\mathrm{L}_{22} \\
(\mathrm{~mm})\end{array}$ & $\begin{array}{l}\mathrm{W}_{\mathrm{s} 2} \\
(\mathrm{~mm})\end{array}$ & $\begin{array}{l}\mathrm{L}_{\mathrm{s} 2} \\
(\mathrm{~mm})\end{array}$ \\
\hline 2.41 & 4.42 & 1 & 7.72 & 0.91 & 1 & 1.27 & 3.63 & 1 & 1.71 & 0.98 \\
\hline
\end{tabular}

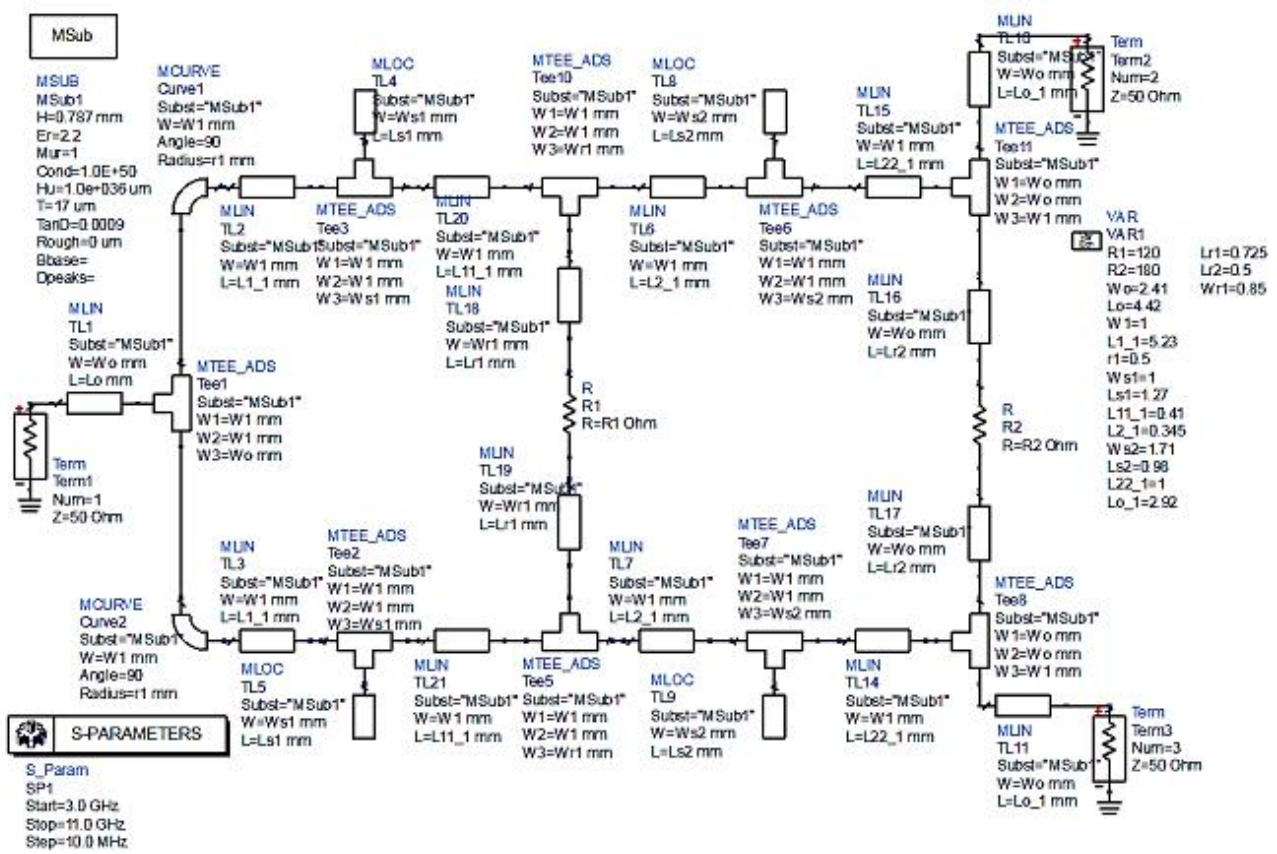

Fig. 12. The microstrip schematic diagram for the proposed splitter. 
The generated layout shown in Fig. 13 is simulated using EM simulator (ADS2011.10). The EM simulator ignores lumped element effect during simulation; it only simulates its footprint. In order to overcome this problem, the generated EM model for the proposed splitter is placed back in the schematic window then the isolation resistors are added and the whole design is simulated again with circuit simulation as shown in Fig. 14.

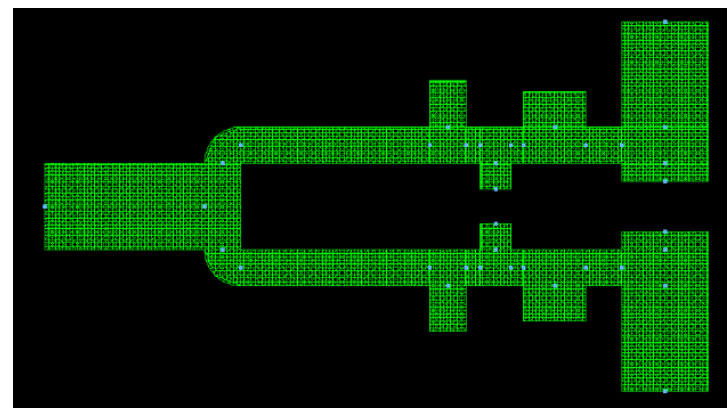

Fig. 13. Generated layout after EM-Simulation.

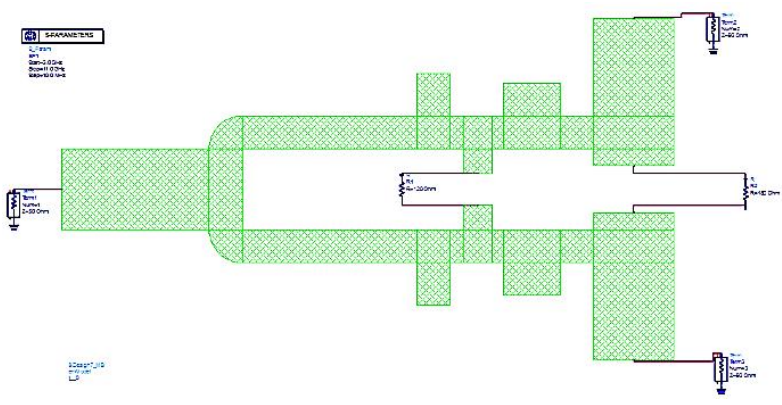

Fig. 14. EM-model with isolation resistors.

The EM Co-Simulations for the proposed splitter are shown in Fig. 15 and Fig.16. The designed splitter achieves an input return loss, $\mathrm{S}_{11}$ is better than $12.2 \mathrm{~dB}$, an output return loss, $\mathrm{S}_{22}$ is better than $19.59 \mathrm{~dB}$, output ports isolations, $S_{23}$ and $S_{32}$ are better than $12.75 \mathrm{~dB}$ and insertion loss, $S_{21}$ and $S_{31}$ are ranges from 3.15 to $3.41 \mathrm{~dB}$ through the whole UWB range. The power splitter has a compact size of $18.38 * 10.25 \mathrm{~mm}^{2}$. Table 3 shows a comparative study for this work with other similar UWB power dividers.

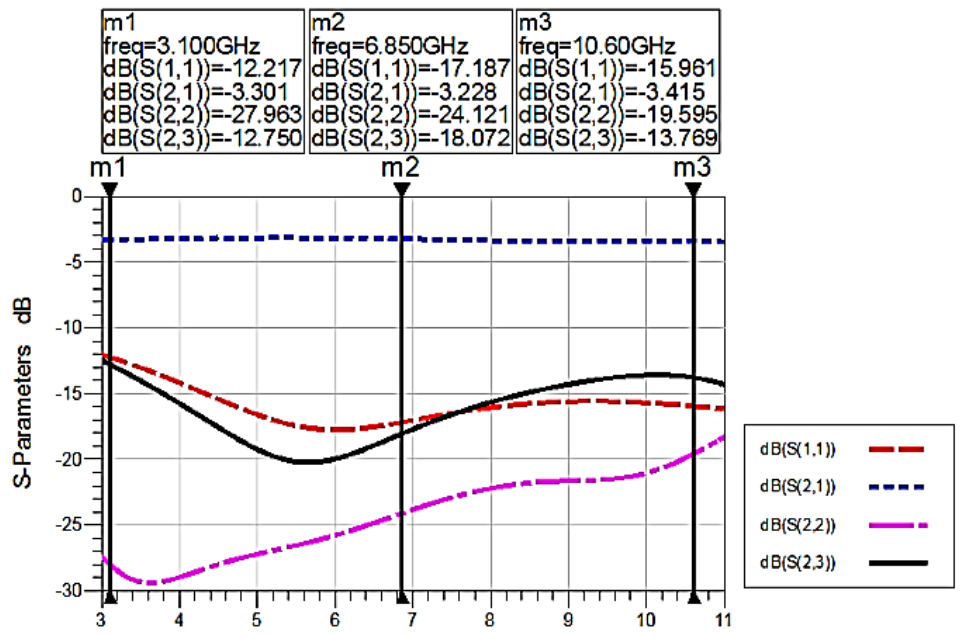

Fig. 15. EM Co-Simulation S-Parameters for the proposed splitter.

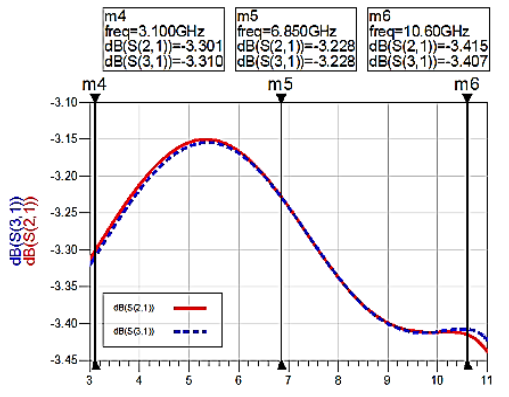

(a)

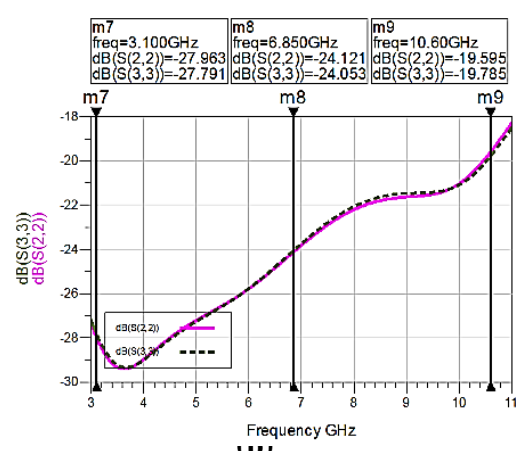

Freq

Fig. 16. (a) Insertion loss S21 and S31, (b) Output matching S22 and S33. 
Table 3. A Comparative Study for this proposed power splitter with other published power dividers.

\begin{tabular}{|c|c|c|c|c|c|}
\hline & This Work & [13] & [7] & [16] & [18] \\
\hline$S_{11 \mathrm{~dB}}$ & 12.2 & 13 & 10 & 10 & 10 \\
\hline$S_{21 \mathrm{~dB}}$ & $3.15-3.41$ & $3 \pm 0.5$ & 3 & around 3 & 3 \\
\hline$S_{22 \mathrm{~dB}}$ & 19.59 & 15 & 15 & 10 & around 6 \\
\hline$S_{23 \mathrm{~dB}}$ & 12.75 & 10 & 10 & 11 & 10 \\
\hline Freq. GHz & $3.1-10.6$ & $3.1-10.6$ & $3.1-10.6$ & $3.1-10.6$ & $2.9-10.9$ \\
\hline Area ${ }^{2}{ }^{2}$ & $18.38 * 10.25$ & $22 * 19.6$ & $18 * 13$ & $\begin{array}{l}\text { around } \\
20 * 45\end{array}$ & $34 * 24$ \\
\hline $\begin{array}{l}\text { substrate } \varepsilon_{r}, \\
\text { thickness } \mathrm{mm}\end{array}$ & $2.2,0.787$ & $2.2,0.787$ & $3.38,0.508$ & $2.2,0.787$ & $2.2,1.0$ \\
\hline Notes & $\begin{array}{c}\text { EM Co- } \\
\text { Simulated }\end{array}$ & Calculated & Measured & Measured & Measured \\
\hline $\begin{array}{c}\text { Design } \\
\text { Methodology }\end{array}$ & $\begin{array}{l}\text { Two section } \\
\text { WPS each } \\
\text { section with a } \\
\text { single stub- } \\
\text { matching } \\
\text { network and } \\
\text { one isolation } \\
\text { resistor. }\end{array}$ & $\begin{array}{l}\text { Single section } \\
\text { WPS with a } \\
\text { single stub- } \\
\text { matching } \\
\text { network. }\end{array}$ & $\begin{array}{l}\text { Single section } \\
\text { WPD with delta } \\
\text { stub and single } \\
\text { isolation } \\
\text { resistor. }\end{array}$ & $\begin{array}{l}\text { Two section } \\
\text { WPD with three } \\
\text { open stubs, two } \\
\text { aperture backed } \\
\text { interdigital- } \\
\text { coupled lines } \\
\text { and two } \\
\text { isolation } \\
\text { resistors. }\end{array}$ & $\begin{array}{c}\text { Single section } \\
\text { unequal WPD } \\
\text { with two folded } \\
\text { shunt } \lambda / 4 \text { short } \\
\text { circuit stubs and } \\
\text { single isolation } \\
\text { resistor. }\end{array}$ \\
\hline
\end{tabular}

\section{CONCLUSION}

This paper represents an UWB modified Wilkinson power splitter; the splitter consists of two sections of unequal electrical length and each with a single open stub and the whole splitter uses two resistors. A detailed analytical solution for the proposed splitter is discussed to obtain exact closed-form design equations. These equations are used to get an initial numerical design parameters for the splitter. These initial design parameters are optimized and improved with the aid of ADS simulator to get the final dimensions of the splitter. The splitter has a compact size, good matching, equal division ratio and the desired bandwidth achieved. Moreover, the splitter achieves better ports isolation in comparison with other similar power dividers that use stubs and for the same UWB frequency range. The proposed splitter is a good candidate for most of Microwave Integrated Circuits because of its improved Sparameters, compact size and simple layout. The analytical solution for the splitter can be generalized to get closed form expressions for $\mathrm{N}$-ways power divider.

\section{REFERENCES}

[1] E. J. Wilkinson, "An N-way Hybrid Power Divider", IRE Trans. Microwave Theory and Techniques, Vol. MTT-8, pp. 116-118, January 1960.

[2] Pozar, D., Microwave Engineering, 3rd edition, Wiley, New York, 2005.

[3] Cohn, S. B., "A Class of Broadband Three-Port TEM-Mode Hybrid," IEEE Trans. Microwave Theory and Techniques, Vol. 19, No. 2, 110-116, 1968.

[4] C.-T. Chiang and B.-K. Chung, "Ultra Wideband Power Divider using Tapered Line," Progress in Electromagnetics Research, Vol. 106, 61-73, 2010.

[5] L. Chang, C. Liao, L.-L. Chen, W. B. Lin, X. Zheng, and Y.-L. Wu, "Design of an Ultra-Wideband Power Divider via the Coarse-Grained Parallel Micro- Genetic Algorithm," Progress In Electromagnetics Research, Vol. 124, 425-440, 2012.

[6] Sai Wai Wong and Lei Zhu, "Ultra-Wideband Power Divider with Good In-Band Splitting and Isolation Performances," IEEE microwave and wireless components letters, VOL. 18, NO. 8, August 2008.

[7] B. Zhou, H. Wang, and W.-X. Sheng, "A Modified UWB Wilkinson Power Divider using Delta Stub,” Progress In Electromagnetics Research Letters, Vol. 19, 49-55, 2010. 
[8] Ali Reza Hazeri, “An Ultra-wideband Wilkinson Power Divider," International Journal of Electronics Vol. 99, No. 4, pp. 575-584,2012.

[9] L. Guo, A. Abbosh and H. Zhu, "Ultra-Wideband In-Phase Power Divider using Stepped-Impedance Three-Line Coupled Structure and Microstrip-to-Slotline Transitions", Electronics Letters 27th February 2014 Vol. 50 No. 5 pp. 383-384.

[10] B. Mishra, A. Rahman, S. Shaw, M. Mohd., S. Mondal and P. P. Sarkar, "Design of an Ultra-wideband Wilkinson Power Divider," The 2014 First International Conference on Automation, Control, Energy and Systems (ACES).

[11] Xiaolong Wang, Iwata Sakagami, Atsushi Mase, and Makoto Ichimura, "Wilkinson Power Divider with Complex Isolation Component and Its Miniaturization," IEEE Transactions on Microwave Theory and Techniques, Vol. 62, NO. 3, March 2014.

[12] Y. Wu, Y. Liu and X. Liu, "Dual-Frequency Power Divider with Isolation Stubs," Electronics Letters, 20 th November 2008 Vol. 44 No. 24.

[13] O. M. H. Ahmed and A. R. Sebak, "Experimental Investigation of New Ultra Wideband In-Phase and Quadrature-Phase Power Splitters," Journal of Electromagnetic Waves and Applications, Vol. 23, No.17-18, 2009.

[14] Osama Ahmed and A. R. Sebak," A Modified Wilkinson Power Divider/Combiner for Ultrawideband Communications," Antennas and Propagation Society International Symposium, APSURSI, 2009.

[15] TaeGyu Kim, Byungje Lee, and Myun-Joo Park, "Dual-Band Unequal Wilkinson Power Divider with Reduced Length", Microwave And Optical Technology Letters, Vol. 52, No. 5 May 2010.

[16] Wei-Qiang Liu, Feng Wei, Chun-Hui Pang, Xiao-Wei Shi, "Design of a Compact UltraWideband Power Divider," The 2012 International Conference on Microwave and Millimeter Wave Technology (ICMMT), Vol. 2, 2012.

[17] J. Reed and G. J. Wheeler, "A Method of Analysis of Symmetrical Four-Port Networks," IRE Trans. Microwave Theory and Techniques, Vol. MTT-4, pp. 246-252, October 1956.

[18] Feng Wei, Xiao Wei Shi and Pei Yuan Qin, Y. Jay Guo "Compact UWB Power Divider with Unequal Distribution Ratio," The 2014 International Workshop on Antenna Technology, IEEE 2014. 